\title{
Which processes shape stellar population gradients of massive galaxies at large radii?
}

\author{
Michaela Hirschmann
}

\author{
UPMC-CNRS, UMR7095, Institut d' Astrophysique de Paris, F-75014 Paris, France
} email: hirschma@iap.fr

\begin{abstract}
We investigate the differential impact of physical mechanisms, mergers (stellar accretion) and internal energetic phenomena, on the evolution of stellar population gradients in massive, present-day galaxies employing a set of high-resolution, cosmological zoom simulations. We demonstrate that negative metallicity and color gradients at large radii ( $>2$ Reff) originate from the accretion of metal-poor stellar systems. At larger radii, galaxies become typically more dominated by stars accreted from satellite galaxies in major and minor mergers. However, only strong galactic winds can sufficiently reduce the metallicity content of the accreted stars to realistically steepen the outer metallicity and colour gradients in agreement with present-day observations. In contrast, the gradients of the models without winds are inconsistent with observations (too flat). In the wind model, colour and metallicity gradients are significantly steeper for systems which have accreted stars in minor mergers, while galaxies with major mergers have relatively flat gradients, confirming previous results. This analysis greatly highlights the importance of both energetic processes and merger events for stellar population properties of massive galaxies at large radii. Our results are expected to significantly contribute to the interpretation of current and up-coming IFU surveys (like MaNGA and Califa), which in turn can help to better constrain still uncertain models for energetic processes in simulations.
\end{abstract}

Keywords. methods: numerical, galaxies: evolution, galaxies: formation, galaxies: elliptical and lenticular, galaxies: stellar content

\section{Introduction}

It is a natural prediction of modern hierarchical cosmological models that the assembly of massive galaxies involves major and minor mergers although most stars in most galaxies have been made in-situ from accreted or recycled gas. Nonetheless, these mergers are expected to play a significant role for the structural and morphological evolution of the massive early-type galaxy population. One important structural galaxy property, which is thought to be strongly influenced by mergers, are the (in general negative) metallicity gradients observed early-on in massive, present-day early-type (e.g. McClure \& Racine 1969), but also in late-type galaxies (e.g. Wyse \& Silk 1989), typically within $1 R_{\text {eff }}$. Thanks to improved and more elaborated observational techniques, present-day metallicity gradients can nowadays be measured out to much larger radii, partly out to $8 R_{\text {eff }}$, (e.g. La Barbera et al. 2012) and even beyond reaching e.g. $\sim 25 R_{\text {eff }}$ in a few nearby galaxies like NGC 5128 or M31 (e.g. Rejkuba 2014; Gilbert et al. 2014).

Previous studies (e.g. Kobayashi 2004) only investigate the emergence of inner gradients (up to $3 R_{\text {eff }}$ ) at comparably poor spatial resolution. Here, we focus on the stellar accretion origin of metallicity \& color gradients in high-resolution re-simulated massive galaxies at large radii $\left(2 R_{\mathrm{eff}}<r<6 R_{\mathrm{eff}}\right)$ in a full cosmological context. We particularly intend to explore the combined effect of energetic phenomena such as strong galactic, stellar-driven winds (and AGN feedback), and of the individual merger and accretion his- 

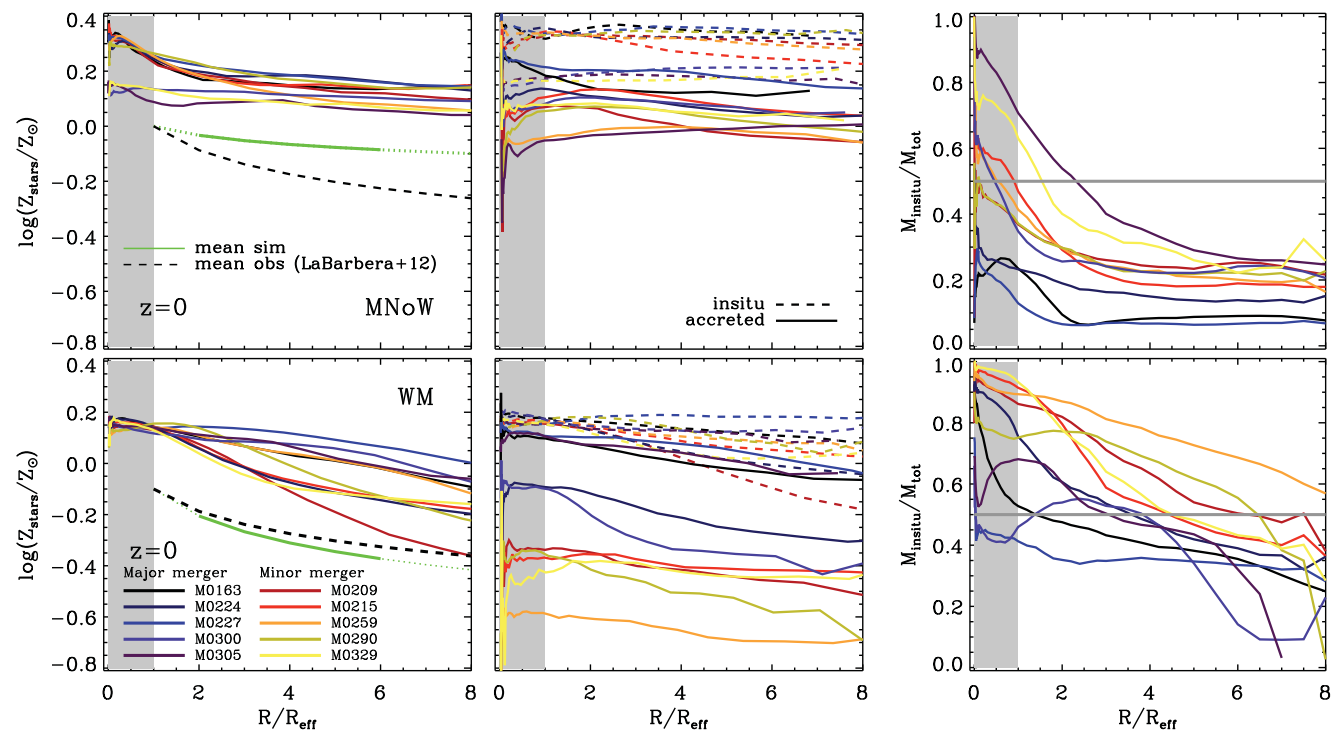

Figure 1. Left column: Total stellar metallicity gradients (mass weighted) at $z=0$ for the ten main galaxies (different colors) simulated with the $\mathrm{MNoW}$ and $\mathrm{WM}$ model. The green solid lines indicate the average gradient at $2<R / R_{\text {eff }}<6$. Middle panels: Metallicity gradients at $z=0$ separated into stars formed in-situ (dashed lines) and accreted stars (solid lines). Right panels: Fraction of in-situ to total stellar mass as a function of radius. Figure taken from Hirschmann et al. (2015).

tories on the in-situ formed and accreted stellar fractions and on steepening or flattening of stellar population gradients at these large radii (Hirschmann et al. 2015).

\section{High-resolution simulations of individual galaxy halos}

We consider the 10 most massive halos (covering a mass range of $6 \times 10^{12}<M_{\text {halo }}<$ $\left.2 \times 10^{13} M_{\odot}\right)$ of our high-resolution, cosmological zoom simulation sets presented in (Hirschmann et al. 2013) and (Hirschmann et al. 2015). The simulations include a treatment for metal enrichment (SNII, SNIa and AGB stars) and a phenomenological feedback scheme for galactic winds (Oppenheimer \& Dave 2006, 2008). The dark matter/gas particles have a mass resolution of $m_{\mathrm{dm}}=2.5 \cdot 10^{7} M_{\odot} h^{-1}$ and $m_{\mathrm{gas}}=m_{\mathrm{star}}=4.2 \cdot 10^{6} M_{\odot} h^{-1}$, respectively with a co-moving gravitational softening length for the gas and star particles of $400 h^{-1} \mathrm{pc}$ and for the dark matter particles of $890 h^{-1} \mathrm{pc}$ (Oser et al. 2010).

These cosmological zoom simulations were shown to be successful in suppressing early star formation at $z>1$, in predicting reasonable star formation histories for galaxies in present day halos of $\sim 10^{12} M_{\odot}$, in producing galaxies with high cold gas fractions (30 - 60 per cent) at high redshift, and in significantly reducing the baryon conversion efficiencies for halos $\left(M_{\text {halo }}<10^{12} M_{\odot}\right)$ at all redshifts in overall good agreement with observational constraints. Due to the delayed onset of star formation in the wind models, the metal enrichment of gas and stars is delayed and is also found to agree well with observational constraints.

\section{Metallicity gradients of present-day massive galaxies}

In Fig. 1, we show the total (mass-weighted) stellar metallicity gradients out to $8 R_{\text {eff }}$ for the main galaxies at $z=0$ in the MNoW and the WM model (as indicated in the legend). For the MNoW galaxies, the (most) central metallicity has values of $Z / Z_{\odot} \sim 0.4$ 


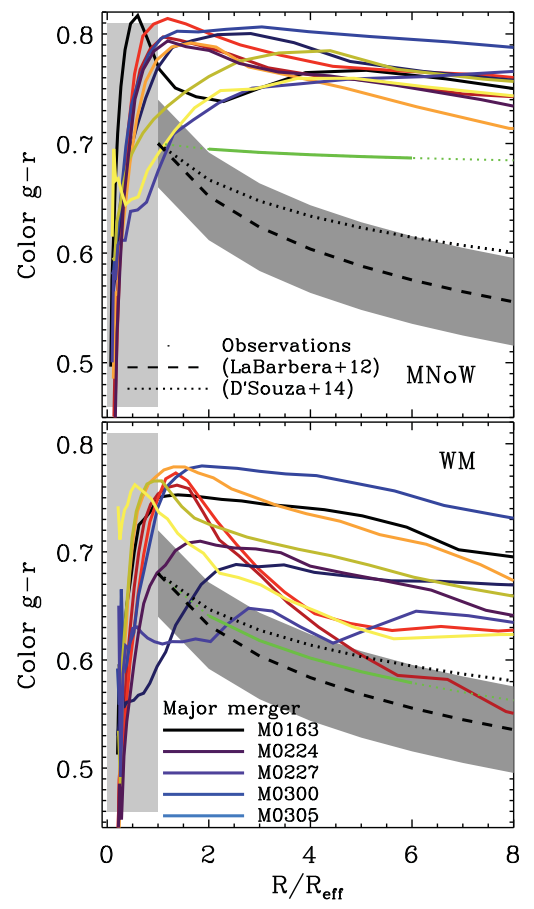

Figure 2. G-r color gradients (mass weighted) at $z=0$ for the ten galaxies (different colors) in the MNoW (top panel) and WM model (bottom panel). The average simulated gradient at $2<R / R_{\text {eff }}<6$ (green solid lines) is compared to observations from La Barbera et al. (2012) and D'Souza et al. (2014). Figure taken from Hirschmann et al. (2015).

and drops to $Z / Z_{\odot} \sim 0.1$ at large radii, the slopes reach a minimum value of -0.25 dex/dex. This gradient is mainly driven by the accreted stars (the in-situ distributions are almost all nearly flat), which have on average roughly solar metallicity (top middle panel of Fig. 1) and dominate most systems outside $1 R_{\text {eff }}$ (top right panel of Fig. 1).

The WM galaxies (bottom panels of Fig. 1) have lower central metallicities $\left(Z / Z_{\odot} \sim 0.2\right)$ with much steeper outer gradients down to $-0.76 \mathrm{dex} / \mathrm{dex}$ with a mean of $-0.35 \mathrm{dex} / \mathrm{dex}$. The reason for the steeper gradients in the WM compared to the MNoW model is twofold: on the one hand, the steeper gradients originate from the accretion of metal-poorer stellar populations. On the other hand, also the in-situ components show metallicity gradients contributing to the overall gradients. The latter is most likely due to infall of (particularly re-infall of previously ejected) metal-poor gas onto the galaxy which can be then turned into metal-poor stars as a consequence of an inside-out growth, the same process causing the metallicity gradients in disk galaxies. Late re-accretion of previously ejected gas occurs typically in the WM model due to the strong galactic winds, but not in the MNoW model, where the in-situ gradients are, therefore, relatively flat (see top middle panel of Fig. 1). However, despite of the partly negative in-situ gradients, we find that on average, the in-situ gradients are reduced by $\sim 0.2$ dex due to accretion of metal-poor stellar populations.

\section{Color gradients of massive, early-type galaxies}

An important set of observables for galaxies are their colours, which are observationally more easy to measure (from images) than age and metallicity (requiring spectroscopic data). Colours are (degenerately) dependent on the intrinsic metallicity and ages of their 

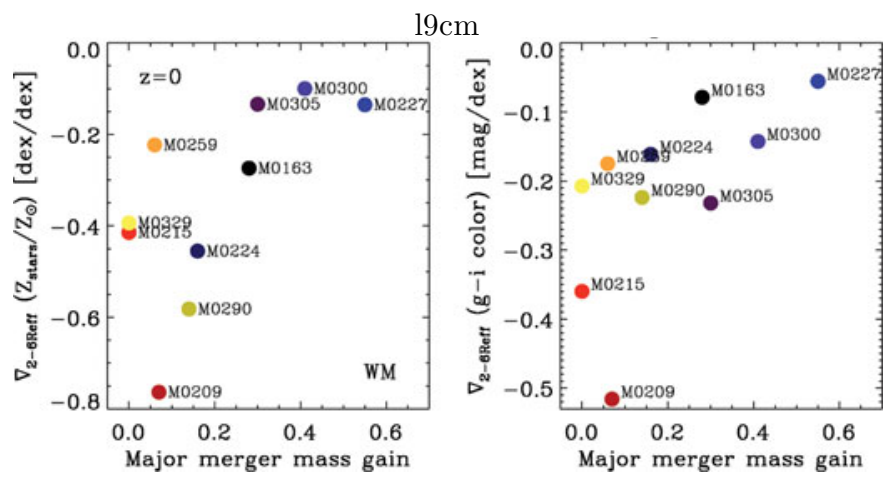

Figure 3. Stellar metallicity (left panel) and g-i color (right panel) gradients (between $2-6 R_{\text {eff }}$ ) versus the mass gain through major mergers for the ten re-simulated galaxies in the WM simulations. The higher the major merger mass gain, the smaller is the slope of the metallicity and color gradient. Figure taken from Hirschmann et al. (2015).

stellar populations. To facilitate a comparison of the simulated radial distributions of galaxy colours to present-day, observed (outer) color gradients we use the metal- and age-dependent models for the spectral evolution of stellar populations of Bruzual \& Charlot (2003), assuming a Chabrier IMF to compute some photometric properties (g-i, $\mathrm{u}-\mathrm{g}$ and g-r colours) of our simulated galaxies.

Fig. 2 shows the the g-r colour gradients (solid lines) for the MNoW and the WM galaxies (top and bottom panel, respectively) at $z=0$. WM galaxies are generally slightly bluer than MNoW galaxies as a consequence of their younger stellar populations with lower metallicity. In addition, all galaxies of both models are significantly bluer at the centre, revealing steep positive colour gradients at $<1 R_{\text {eff }}$ which is an unrealistic artefact of our models most likely due to missing AGN feedback resulting in too young central stellar populations.

At larger radii ( $>2 R_{\text {eff }}$ ), the two models behave differently. The MNoW galaxies have only very shallow colour gradients, either slightly decreasing or increasing with an average slope of $-0.02 \mathrm{mag} / \mathrm{dex}$ for the g-r colors. The shallow colour gradients in the MNoW model stem from the relatively flat age and only slightly negative metallicity gradients. The former originates from a relatively old in-situ stellar population (compared to the accreted stars), and the latter from the accretion of relatively metal-rich stellar systems in the MNoW model. Instead with galactic winds, nearly all of the WM galaxies become continuously bluer with increasing radius and thus, reveal significantly steeper negative colour gradients than the MNoW galaxies (with mean slopes of $-0.13 \mathrm{mag} / \mathrm{dex}$ for the g-r colours). This behaviour is entirely driven by the steeper metallicity gradients of the WM galaxies washing out any effect of the slightly increasing age gradients. Note that we find qualitatively similar results for the g-i and $\mathrm{u}$-g color gradients (see Hirschmann et al. 2015).

\section{The impact of the galaxy merger history}

The black-blue lines in Fig. 1 and 2 indicate galaxies which experienced at least one major galaxy merger since $z=2$, while the red-yellow lines illustrate those having undergone only minor galaxy mergers. Present-day galaxies having experienced a recent major merger have typically flatter gradients than those with a more quiet merger history.

Fig. 3 quantifies the connection between the galaxy merger history and the steepness of the metallicity and color gradients: we show the fitted metallicity (left panel) and color 
gradients (right panel) at $z=0$ for WM galaxies versus the mass gain by major mergers. The mass gain by major mergers considers the entire stellar mass which was brought into the main galaxy by major mergers since $z=2$ normalised to the present-day stellar mass.

Both the fitted metallicity and color gradients correlate with the past merger history: for a major merger mass gain above $20 \%$, the metallicity (color) gradients are flatter than $-0.3(-0.2)$ dex/dex. Instead, for lower x-values, the metallicity (color) gradients are mostly more negative than $-0.4(-0.2) \mathrm{dex} / \mathrm{dex}$. This is due to the fact that the accreted stars show a huge variety of metallicities from $Z / Z_{\odot} \sim-0.6$ to $Z / Z_{\odot} \sim+0.1$ (see bottom middle panel of Fig. 1) depending on the exact merger history: in case of a recent major merger, the accreted metallicity is significantly larger than without a major merger (as more massive galaxies have higher metallicity). The higher metallicity of the accreted stars together with the different mixing behaviour (violent relaxation) in case of major mergers flattens the total metallicity gradients. The present-day color gradients are entirely driven by the behaviour of the metallicity gradients, leading to the same behaviour.

This result bears an important implication for observations, as it can help to reconstruct the past assembly history for observed present-day metallicity and color gradients: The relation between the steepness of the gradients and the individual merger history implies that observed massive galaxies having steep outer gradients most likely have not experienced any major merger event after $z=1$, but instead several minor mergers.

\section{Comparison to observations}

Starting with the colour gradients, in the photometric study by La Barbera et al. (2012), they investigated colour gradients of early-type galaxies $\left(M_{\text {stellar }}>3 \times 10^{10} M_{\odot}\right)$ even out to $8 \times R_{\text {eff }}$ using the SDSS-based Spider survey. They measure average slopes for g-r colours of $-0.16 \pm 0.04 \mathrm{mag} / \mathrm{dex}$ (see black dashed line and grey shaded areas in Fig. 2). Compared to observed, g-r colour gradients, MNoW galaxies have on average too flat gradients $(-0.02 \mathrm{mag} / \mathrm{dex})$, while those of the WM galaxies are reasonably steep $(-0.13 \mathrm{mag} / \mathrm{dex})-$ in good agreement with the observations.

The recent work of D'Souza et al. (2014) show g-r colour profiles out to $100 \mathrm{kpc}$ for stacked ellipticals (to be more precise, high concentration galaxies) with masses between $10^{10}-10^{11.4} M_{\odot}$ using roughly 45.500 galaxies from the SDSS survey. They find values between -0.11 and $0.14 \mathrm{mag} / \mathrm{dex}$ with little mass trend, in perfect agreement with the g-r colour gradients predicted by our simulations (see black dotted lines in Fig. 2, where we have used the slope of $-0.11 \mathrm{mag} / \mathrm{dex}$ measured for stellar masses of $10^{11.2} M_{\odot}$ ).

Turning to metallicity gradients, a recent study of Pastorello et al. (2014), using the SLUGGS survey, investigates metallicity gradients at least out to $2.5 R_{\text {eff }}$. For comparable stellar masses, their galaxies reveal slopes between -1.15 and $+0.18 \mathrm{dex} / \mathrm{dex}$. In addition, in the study by La Barbera et al. (2012), they derive metallicity gradients of their sample of early-type galaxies. For massive galaxies with $10^{11}<M_{\text {stellar }}<7 \times 10^{11} M_{\odot}$ they find outer metallicity gradients $\left(1-8 \times R_{\text {eff }}\right)$ in the range of -0.29 to $-0.74 \mathrm{dex} / \mathrm{dex}$ depending on the stellar population model (illustrated by the black dashed lines in Fig 1).

Overall, for both colors and metallicities, our WM galaxies are able to cover such a broad range of slopes (e.g. from -0.8 to $-0.1 \mathrm{dex} / \mathrm{dex}$ for metallicity) much better than the MNoW galaxies whose slopes are on average too flat (e.g. between -0.25 and $+0.03 \mathrm{dex} /$ dex for metallicity). The average metallicity and color gradient of the WM galaxies is in excellent agreement with the ones of La Barbera et al. (2012). As expected 
a priori, this implies that a strong stellar feedback is a key mechanism to be consistent with observed steep metallicity gradients in massive galaxies in the local Universe.

\section{Conclusions}

Analysing zoom simulations of 10 massive galaxies suggests that the outer negative metallicity and color gradients (at radii $r>2 R_{\text {eff }}$ ) of present-day massive galaxies are mainly determined by the accretion of stars with lower metallicity (see Hirschmann et al. 2015). Towards low redshift, stars accreted in low metallicity galaxies become more and more dominant at large radii and the metallicity gradients of in-situ formed stars in the wind model are enhanced by $\sim 0.2 \mathrm{dex} /$ dex by accretion of metal-poor systems. The model with galactic winds predicts steeper total metallicity gradients (on average $-0.35 \mathrm{dex} / \mathrm{dex}$ ) as the accreted stellar systems are significantly more metal-poor and despite of the fact that much less stellar mass in total is accreted compared to the no-wind model. Note that the negative outer color gradients in our simulated presentday massive galaxies are entirely driven by the negative outer metallicity gradients, and therefore, originate from the same processes, the interplay of strong stellar feedback and the individual merger history.

Even if not explicitly discussed in this article, first, preliminary simulations including AGN-driven winds indicate that this additional process does qualitatively not alter the stellar accretion origin of metallicity gradients at large radii, but apparently mainly affects the inner metallicity gradients.

Overall, we can conclude that a combination of stellar accretion (in minor mergers) of low mass satellites (as predicted from idealized experiments by Villumsen 1983) and strong stellar feedback results in steep outer metallicity gradients successfully matching the broad range of observed metallicity profiles of local galaxies at large radii.

\section{Acknowledgements}

MH acknowledges financial support from the European Research Council via an Advanced Grant under grant agreement no. 321323NEOGAL.

\section{References}

Bruzual M. \& Charlot 2003, MNRAS, 344, 1000

D'Souza R. et al. 2014, MNRAS, 443, 1433

Gilbert K. et al. 2014, ApJ, 796, 20

Hirschmann M. et al. 2013, MNRAS, 436, 2929

Hirschmann M. et al. 2015, 449, 528

Kobayashi C., 2004, MNRAS, 347, 740

La Barbera F. et al. 2012, MNRAS, 426, 2300

McClure R. \& Racine R. 1969, AJ, 74, 1000

Oppenheimer B. \& Dave R. 2006, MNRAS, 373, 1265

Oppenheimer B. \& Dave R. 2008, MNRAS, 387, 577

Oser L. et al. 2010, ApJ, 725, 2312

Pastorello N. et al. 2014, MNRAS, 442, 1003

Rejkuba M. et al. 2014, ApJL, 791, 6

Villumsen J. 1983, MNRAS, 204, 219

Wyse R. \& Silk J. 1989, ApJ, 339, 700 